\title{
Some design guidelines to adapt a Darrieus vertical axis turbine for use in hydrokinetic applications
}

\author{
Francesco Balduzzi ${ }^{1}$, Pier Francesco Melani ${ }^{1}$, Giuseppe Soraperra ${ }^{2}$, Alessandra Brighenti $^{2}$, \\ Lorenzo Battisti ${ }^{3}$, and Alessandro Bianchini ${ }^{1, *}$ \\ ${ }^{1}$ Department of Industrial Engineering (DIEF), Università degli Studi di Firenze, Via di Santa \\ Marta 3, 50139 Firenze, Italy \\ ${ }^{2}$ HE-PowerGreen s.r.l., Via Solteri 74, 38121 Trento, Italy \\ ${ }^{3}$ Department of Civil, Environmental and Mechanical Engineering (DICAM), Università di Trento, \\ Via Mesiano 7, 38123 Trento, Italy
}

\begin{abstract}
The use of vertical-axis turbines is raising interest in the field of hydropower production from rivers or water channels, where suitable mass flows are available, without the need of high water jumps or large construction sites. Although many optimization studies on vertical-axis turbines have been carried out for wind applications, lesser examples exist in the technical literature regarding hydrokinetic turbines. In the latter case, the best trade-off between power output and low structural stress is more dependent on the fluid dynamic loadings rather than the inertial loadings, due to the higher fluid density and lower rotation speed. The present work shows the results of an industrial study case application, in which the design of a traditional three-blade Darrieus rotor has been adapted for operating in water flows via hydrokinetic technology. Some specific design rules will be discussed, showing the different concepts adopted for the machine layout in order to achieve the best efficiency and performance. Multiple geometrical parameters of the rotor configuration were involved during the analysis: the number of rotor blades, i.e. two or three blades, the rotor's shape, i.e. traditional $\mathrm{H}$-shape or unconventional L-shape, and the use of power augmentation systems. The analysis of the numerical results was focused on the following output targets: maximum power coefficient, optimal tip speed ratio (TSR), rotor thrust, blade normal force and the upstream and downstream flow field influence. The outcome of the study shows how the best configuration differs from the common solutions for wind application. Moreover, a high power enhancement can be achieved while guaranteeing a good compromise in terms of structural loads.
\end{abstract}

\section{Introduction}

Traditional hydroelectric power plants exploit the potential energy accumulated by water that has been previously collected inside a storage reservoir. The water is stored thanks to

\footnotetext{
*Corresponding author: alessandro.bianchini@unifi.it
} 
the construction of a dam or a barrier, which force the water to fall from a high height. The energy harvesting depends on available flow rate and the altitude of the water head. In general, this solution requires large investment costs, a suitable area for the plant site and a lot of construction work. Nowadays, hydropower represents almost $20 \%$ of the total worldwide electricity production [1] and, in many nations, most of the favorable locations have been already exploited.

In recent times, the increasing need of alternative energy sources has led to the expansion of hydroelectric power generation to non-conventional sites, such as small natural streams, canals or artificial water networks [2]. For such applications, the technology required for harvesting the energy content is based on hydrokinetic energy conversion systems, which require flow velocities typically higher than $1 \mathrm{~m} / \mathrm{s}$. Unlike traditional hydraulic turbines, the operation principle of hydrokinetic turbines (HKTs) is inherited from the more widespread wind turbines (WTs), which exploit the kinetic energy of the fluid flow for generating electrical power, rather than the potential one, providing the Froude-Mach analogy being satisfied. Therefore, the experience learned in the field of wind turbines is progressively being transferred in order to develop machines capable of working in water flows, as well as marine currents and tides.

Analogously to wind turbines, hydrokinetic turbines can be classified based on the relative orientation of the revolution axis with respect to the flow direction, i.e. horizontal axis and vertical axis turbines. In the latter category, also known as cross-flow turbines, the most common technology is the lift-type Darrieus turbine, where the lift force generated from the water current over a hydrofoil is transformed into the rotation of the blade. The design of Darrieus vertical axis turbines is still not fully assessed even in the wind energy field, because of the complex aerodynamics involved. Their intrinsically unsteady operation in fact, due to the continuous variation of the incidence angle experienced by the blades during one rotor revolution, leads to specific challenges to be faced in order to find the best trade-off between performance optimization and structural integrity. Currently, a global convergence on a standard three-blade $\mathrm{H}$-shaped rotor is being reached, featuring a medium-high solidity $(\sigma=N c / D)$ in order to guarantee suitable chord-based Reynolds number $\left(R e_{c}=\varrho c W / \mu\right)$.

In the hydrokinetic energy field, the need of further research is even more crucial due to the early stage of development. Typical geometrical design features involved in the optimization process are still limited to the basic rotor parameters, such as the hydrofoil shape [4], the number of blades (solidity) [5] and the aspect ratio [6], although a well-defined standard is still far to be assessed. As in the wind energy field, there is also a lack of knowledge regarding the implementation of additional systems for enhancing the performance, represented by active or passive flow control. In particular, various works exist in the technical literature regarding the use of active pitch control $[7,8]$ or external components such as deflectors and ducts [9]. Such devices, although capable of providing good enhancement of performance, lead to a significant increase of the turbine complexity and, therefore, of both the reliability and the cost of installation. Conversely, the use of passive flow control devices to be applied on the surface of hydrofoils, although largely investigated for wind applications, is still not being studied for hydrokinetic cross flow turbines, to the authors' knowledge.

\section{Design considerations \& scope of the activity}

In water applications, the typical flow velocities are lower than those encountered in wind ones, but density is almost three orders of magnitude higher. This leads to operating conditions involving, on the one hand, higher hydrodynamic torque and forces and, on the other hand, lower revolution speed. For instance, at given rotor swept area, a water velocity 
of $0.85 \mathrm{~m} / \mathrm{s}$ is sufficient to provide the same input power of a standard wind velocity of $8 \mathrm{~m} / \mathrm{s}$. As a result, the design of the machine has to take into account different aspects:

- HKTs can harvest more energy than WTs for the same turbine dimensions (from 3 to more than 10 times higher);

- HKTs operate at higher Reynolds (at least 2-3 times higher), thus allowing higher efficiency even if in case of small-scale applications and a lesser need of increasing the chord length (and therefore the solidity) to achieve suitable chord Reynolds number;

- from a structural point of view, the relative weight of various load sources is different, since hydrodynamic forces are more relevant than inertial (centrifugal) forces.

Based on the aforementioned considerations, when dealing with wind turbines it is necessary to limit the optimal TSR, i.e. the rotational speed at which the rotor produces the maximum power at rated wind speed, in order to avoid excessive centrifugal loadings. Indeed, due to the low-density fluid, centrifugal forces have a high impact on the overall loading with respect to aerodynamic forces. Since both the maximum efficiency and the optimal TSR tend to increase as the number of blades is reduced, the optimal trade-off between aerodynamic performance and structural stress is commonly found with a three-blade rotor. The feasibility of a two-blade vertical axis wind turbine (VAWT) rotor is viable only in case of micro turbines or in case of high solidity (i.e. large chord to radius ratio), to limit the peripheral speed of the blade. As an additional consideration concerning the solidity of VAWTs' rotors, high solidities are generally necessary to improve the poor starting torque performance. Conversely, HKTs placed in more constant water flows are less sensitive to the self-starting problem. Moreover, high solidity can be more problematic due to the increase of hydrodynamic loads. Lower solidities can be advantageous also in terms of regulation control system, thanks to a more flat torque characteristic curve around the TSR of maximum efficiency.

The current activity aims at providing some design considerations applied to a preliminary prototype of a three blade Darrieus rotor that has been adapted for operating in water flows by numerically exploring the influence of some geometrical parameters on the expected performance as well as on the blade and turbine loadings. In this scenario, an accurate estimation of hydrodynamic performance is even more critical and the use of standard low-order industrial tools could be detrimental. An error in the estimation of lift and drag characteristics and, in general of the expected forces and power output, could lead to wrong design considerations. High fidelity CFD simulations are therefore pivotal for a better understanding of all fluid dynamic phenomena occurring on the hydrofoil, as well as the wake evolution.

As a first step of the activity, the effect of different rotor arrangements will be shown. When dealing with hydrokinetic turbines in fact, it is possible to seek for the maximum efficiency by reducing the number of blades, at the expense of an increase of the optimal TSR, due to the aforementioned looser constraint on the inertial forces. An additional advantage is provided by the related savings in terms of both weight of the rotor and manufacturing production costs, as well as a reduction of the passive resistant torque due to the drag of the supporting struts. On the other hand, the less balanced layout leads to an increase of loads fluctuations, which must be properly addressed not to affect the life expectancy of the machine.

As a second step, the effect of installing passive flow control device as an easy and cost-effective solution for a fine-tuning of the performance will be shown. In particular, Gurney Flaps (GFs) are small tabs applied to the pressure side of the blade trailing edge to enhance the lift force [10]. The formation of both a stagnation zone upstream of the GF and a pair of counter-rotating vortices downstream the GF leads to an increase of pressure 
difference between suction side and pressure side of the blade, thus determining a substantial increase in the lift coefficient.

According to the technical literature $[11,12]$, it was found that the height of the GF should be kept below the boundary layer thickness in order to obtain a beneficial lift-to-drag ratio. In previous works carried out by some of the authors for VAWTs applications $[13,14]$, it was found that the best performance can be achieved when a GF with a height of $2 \% c$ was applied on the inner side of the airfoil.

\section{Study case}

The analyzed industrial study case started from a baseline configuration of the three-blade rotor equipped with the DU06W200 hydrofoil. The hydrofoil was mounted with outward concavity to enhance the power extraction during the upstream part of the rotor revolution. The main geometrical parameters were first assessed by means of a preliminary investigation with standard low order models, resulting in a straight bladed Darrieus rotor featuring a blade chord $(c)$ of $0.25 \mathrm{~m}$, a rotor diameter $(D)$ of $2.0 \mathrm{~m}$ and a rotor height $(H)$ of $3.0 \mathrm{~m}$. In the baseline configuration, namely $3 B$ in Figure 1.a, the solidity $\sigma$ is 0.375 .

Two modified rotor configurations were then defined:

- a standard two blade H-type rotor, namely $2 B-H$ in Figure 1 .a, where the blades are symmetrically positioned $\left(180^{\circ}\right.$ angle) with respect to the rotation axis. The rotor is split at mid span $(1.5 \mathrm{~m})$ such as the upper and bottomer portions can be positioned perpendicularly to each other, in order to compensate the less balanced design;

- an unconventional two blade L-type rotor, namely $2 B-L$ in Figure 1.a, where the blades are perpendicularly positioned $\left(90^{\circ}\right.$ angle) with respect to the rotation axis. The upper and bottomer portions of the rotor are positioned symmetrically to each other, in order to compensate the less balanced design.

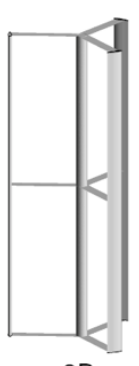

3B

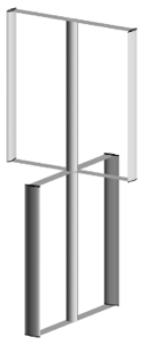

$2 \mathrm{~B}-\mathrm{H}$

(a)

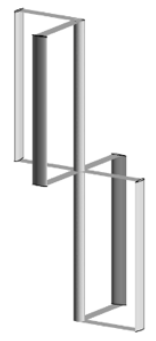

2B-L

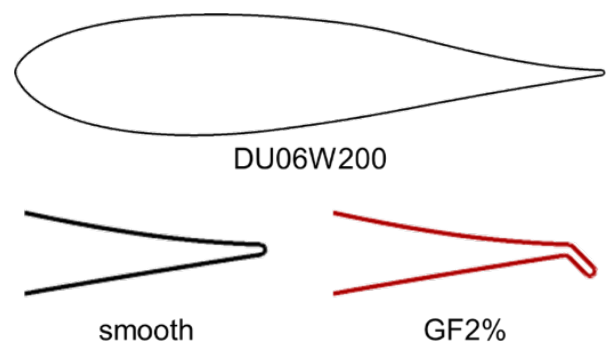

(b)

Fig. 1. (a) rotor configurations, (b) blade configurations.

As will be shown in the results section, the $2 B-H$ configuration was the most promising in terms of expected performance. To further refine the design of such configuration, in order to find the most suitable trade-off between power output and structural loadings, the blade trailing edge was modified by adding a $2 \% c$ Gurney Flap with an inclination of 45 degrees (Figure 1.b). According to [13], such GF arrangement represents the best solution in terms of lift enhancement without a too excessive penalty due to the drag resistance. For all of the two-blade configurations, the chord length of the three-blade rotor was kept constant, therefore the solidity was reduced to 0.25 . 


\section{Methodology}

The numerical investigation was performed by means of two dimensional CFD simulations with the commercial software ANSYS ${ }^{\circledR}$ FLUENT $^{\circledR}$. The computational framework was thoroughly assessed and validated with experimental data for various test cases by some of the authors in previous studies [13-18]. According to previous results, the unsteady Reynolds-Averaged Navier-Stokes (URANS) approach was adopted with the Menter's $\varkappa$ - $\omega$ SST (shear stress transport) model for turbulence closure. The pressure-based formulation was solved by means of the coupled algorithm for the pressure velocity coupling.

A two-dimensional computational domain was used. The rotating domain, enclosing the rotor geometry, is connected to the stationary domain though a sliding mesh interface. The domain dimensions are reported in Figure 2. The undisturbed wind speed $\left(V_{0}\right)$ is imposed at the inlet boundary and the ambient pressure is imposed at the outlet. The settings adopted for the generation of the computational grid were defined according to Refs. [16-18] for the smooth case: the hydrofoil was discretized using 750 nodes on the blade surface for the stable functioning points along the power coefficient $\left(C_{P}\right)$ curve, i.e. TSR higher than the efficiency peak, while the number of nodes was increased to 1500 for low TSRs. In case of hydrofoil equipped with Gurney Flap, a much finer grid was necessary, according to [13-15], therefore a nodes' count on the blade surface was 2000. The resulting size of the different meshes is reported in Table 1, together with the azimuthal timestep adopted for the time advancing of the solution. Figure 2 shows different details of the computational grids: the mesh refinement in the region of the rotor's wake, the mesh refinement around the blade and the boundary layer resolution on the trailing edge, both for the smooth and the GF cases.

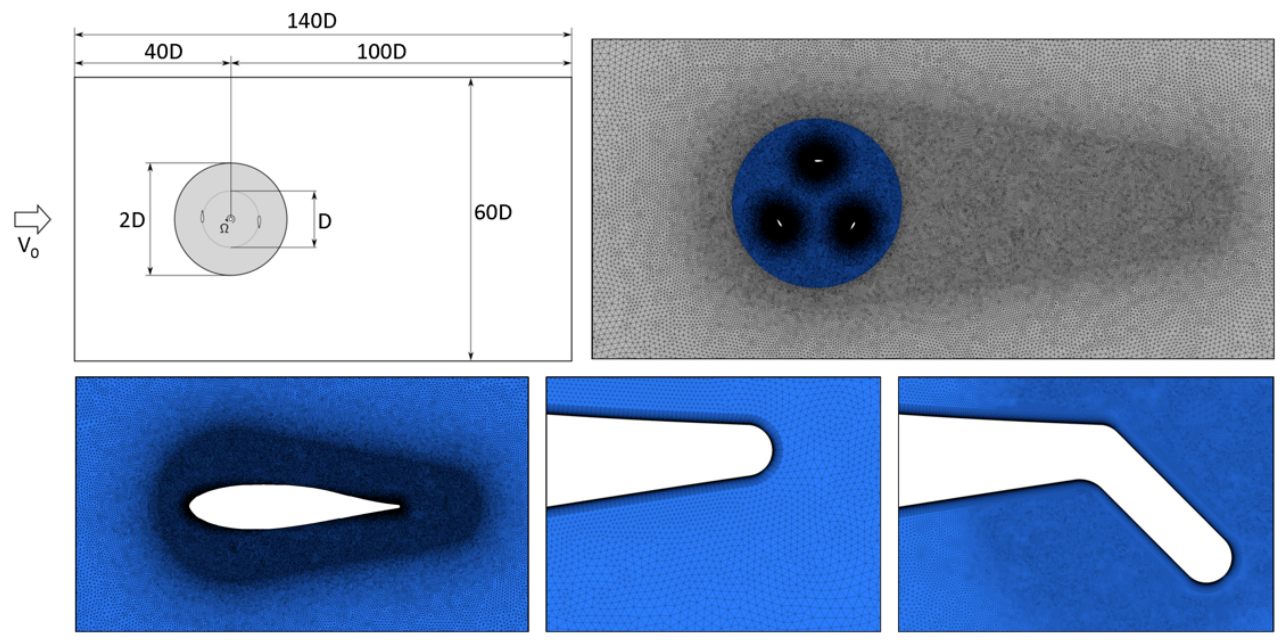

Fig. 2. Computational domain dimensions and different details of the computational grid.

Table 1. Discretization properties.

\begin{tabular}{|c|c|c|c|}
\hline Parameter & 3 blade & 2 blade (smooth) & 2 blade (GF) \\
\hline \# Profile nodes & $750-1500$ & $750-1500$ & 2000 \\
\hline \# Total elements & $0.7 \times 10^{6}-1.4 \times 10^{6}$ & $0.6 \times 10^{6}-0.9 \times 10^{6}$ & $1.3 \times 10^{6}$ \\
\hline Angular timestep [deg] & $0.2-0.4$ & $0.2-0.4$ & 0.1 \\
\hline
\end{tabular}




\section{Results}

In the present section, the main outcomes of the numerical study are analysed by means of hydrodynamic power, torque and forces output, as well as flow field visualization. In particular, forces are reported per unit blade span, while power and torque are reported in dimensionless form in terms of power and torque coefficients, respectively defined as:

$$
\begin{aligned}
& C_{P}=\text { Power } /\left(0.5 \varrho A V_{0}^{3}\right) \\
& C_{T}=\text { Torque }\left(0.5 \varrho A V_{0}^{2} \cdot R\right)
\end{aligned}
$$

where $R$ is the rotor radius and $A$ is the rotor frontal area.

Figure 3.a shows the trends of power curves as a function of the tip-speed-ratio $\left(\mathrm{TSR}=\Omega R / V_{0}\right)$ for all of the tested geometrical configurations, while Figure 3.b shows the increase of power coefficient peak and optimal TSR (i.e. TSR when the maximum power is extracted) achieved with the different $2 B$ configurations with respect to the baseline $3 B$ configuration. From a perusal of the figures, one can readily notice that, as expected, the lower solidity of the two-blade rotors allows reaching higher efficiencies at higher rotating speeds.

The larger power gain is guaranteed by the smooth configurations, since the $2 B-H$ and $2 B-L$ cases show a $13.1 \%$ and $12.6 \%$ increase over the baseline $3 B$ configuration, respectively. On the other hand, such improvement is achieved in both cases at the expenses of reaching an optimal TSR for maximum power extraction roughly $23.5 \%$ higher than the corresponding value for the $3 B$ case. It is worth noting that the $2 B-L$ case shows a much abrupt worsening of the efficiency for TSR lower than the optimal value, thus justifying the choice of testing the performance of blades equipped with GF for the $2 B-H$ case only.

When analysing the predicted power performance of the $2 B-H-G F 2 \%$ case, different considerations can be made. The maximum power coefficient is similar to the corresponding smooth configuration, since the increase is just slightly lower $(+10.7 \%)$, but the entire power curve is shifted toward lower rotating speeds. Indeed, the increase of the optimal TSR is only $11.8 \%$, therefore much closer to the baseline case. It appears that the use of GFs allows achieving a good compromise between maximum efficiency and increase of rotating speed.

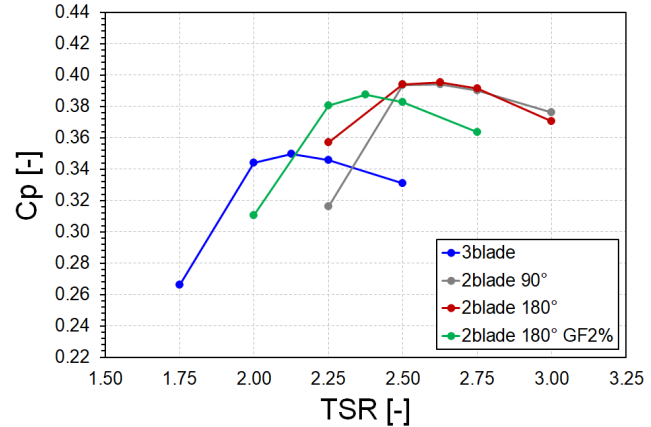

(a)

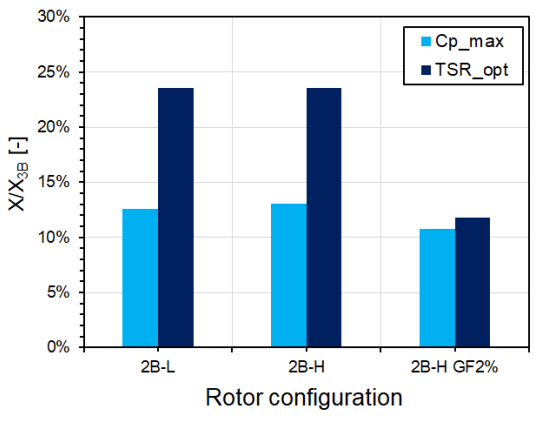

(b)

Fig. 3. (a) power coefficient curves, (b) relative increment of $C_{p, \max }$ and $T S R_{\text {opt }}$ of the two-blade cases with respect to the three-blade case. 
From the analysis of the flow field features reported in Figure 4, some additional observations can be made. The figure shows the vorticity contours in order to appreciate the evolution of the viscous wake of each blade. Only the smooth cases are analyzed, in order to focus the attention on the effect of the rotor arrangement. For each case, four azimuthal positions of the blade are shown, i.e. $0^{\circ}, 90^{\circ}, 120^{\circ}$ and $180^{\circ}$, for the operating condition of maximum $C_{p}$ (different optimal TSR for each specific case). The $3 B$ case contours show that the vorticity generated by the blade during the leeward part of the revolution (third quadrant) is much more intense. Moreover, the lower spacing between the blades forces each blade to operate through high vorticity regions for a larger portion of the rotation. The blades of the $2 B-H$ case are allowed to operate under more favorable conditions, since each blade encounters a much lesser disturbance from the opposite blade. Finally, although the front blade of the $2 B-L$ case operates under ideal conditions, the trailing blade is forced to operate under a particularly disturbed flow.

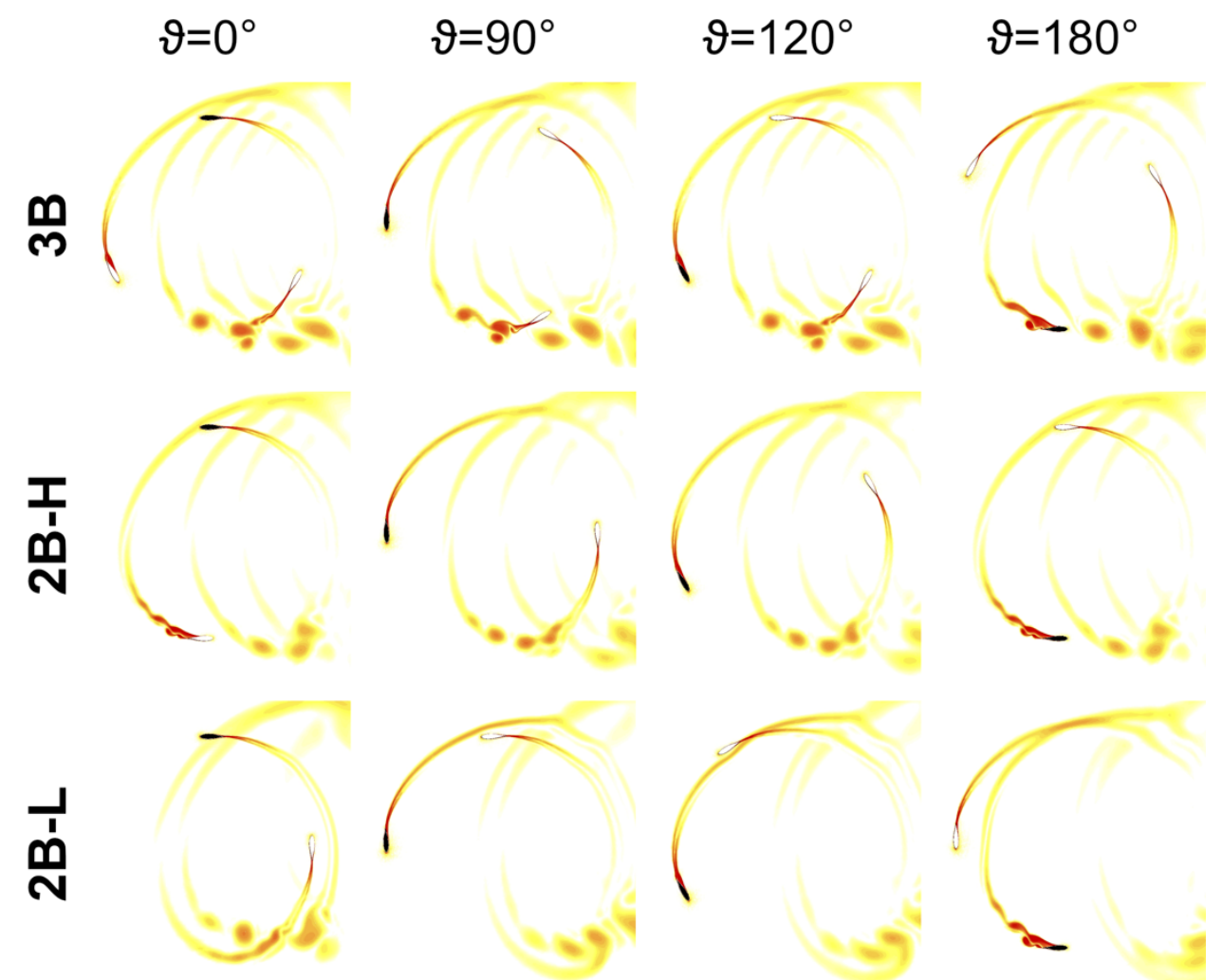

Fig. 4. Vorticity contours at different azimuthal positions for the smooth configurations at $T S R_{o p t}$.

Another significant advantage of the two-blade configurations is that, thanks to the mid-span blade splitting to position the upper and bottomer portions perpendicularly to each other, the overall hydrodynamic loads experienced by the rotor due to the sum of forces of all blades is equivalent to a four-blade rotor. Global axial and lateral force fluctuations are significantly smoothed out in the $2 B-H$ case, as can be noticed from the $F_{x}$ and $F_{y}$ trends reported in Figure 5. The central shaft of the $3 B$ rotor must be dimensioned to resist to strongly fluctuating loading, while the solution adopted for the two-blade configurations allows a substantial balancing of forces. 
Once proven the superior performance of the $2 B$ configurations, the different $2 B$ arrangements were investigated in more detail. Figure 6 shows the trends of torque extracted by a single blade during its rotation for all of the tested $2 B$ cases at each respective optimal TSR. Due to the asymmetric arrangement of the $2 B-L$ case, both the front blade (B1) and the trailing blade (B2) torque coefficients are reported. Although the lower torque output of the trailing blade is compensated by the higher torque output of the front blade, the overall variation of power extraction is negligible. The solution is therefore discarded due to the more unbalanced design.

Focusing the attention on the influence of Gurney Flaps, the $2 \% c$ tab mounted on the inner side of the blade profile allows enhancing the energy extraction in case of positive incidence, i.e. during the downwind half of the revolution. Conversely, the lower lift and greater drag generated in case of negative incidence leads to a reduction of torque output in the upwind half of the revolution.

The main advantage of the GF configuration can be observed in terms of turbine and blade loadings. The overall trends of force magnitude, reported in Figure 7.a, show a strong reduction of loadings acting on the central shaft of rotor, since the mean value for the $2 \mathrm{~B}-\mathrm{H} \mathrm{GF} 2 \%$ case is $14 \%$ lower than the respective smooth case. Such result is pivotal form a structural point of view, since the hydrodynamic forces acting on hydrokinetic turbines are more relevant than the more common corresponding wind turbines. This reduction is primarily due to a better balancing of the lateral force, since the $F_{y}$ trends shown in Figure 7.b reveal that the average value is roughly halved for the $2 B-H G F 2 \%$ case.

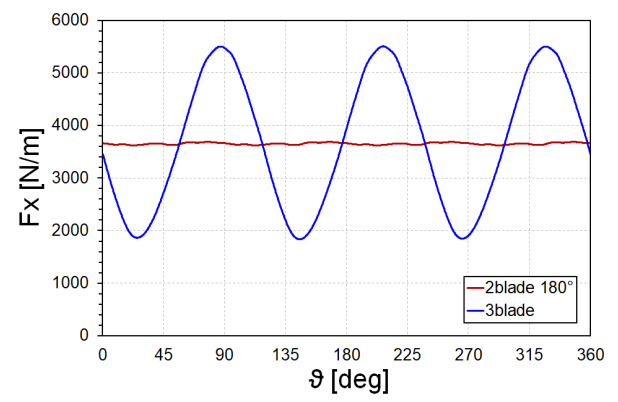

(a)

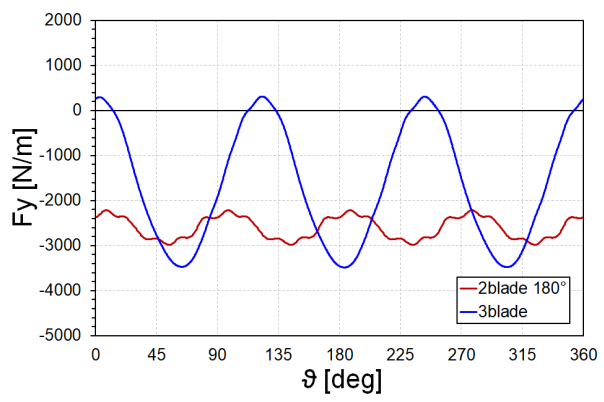

(b)

Fig. 5. (a) global axial force and (b) global lateral force per unit span at $T S R_{o p t}$.

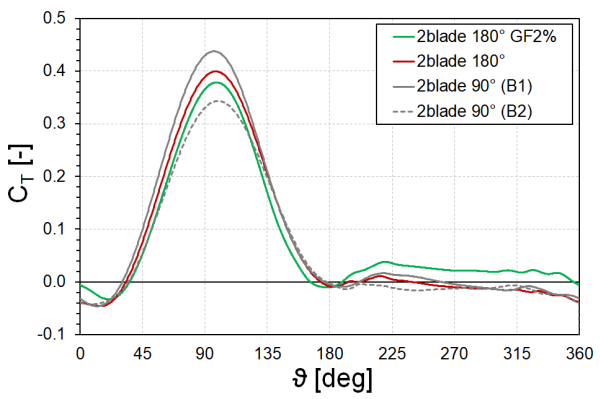

Fig. 6. Torque coefficient of a single blade at $T S R_{\text {opt }}$. 


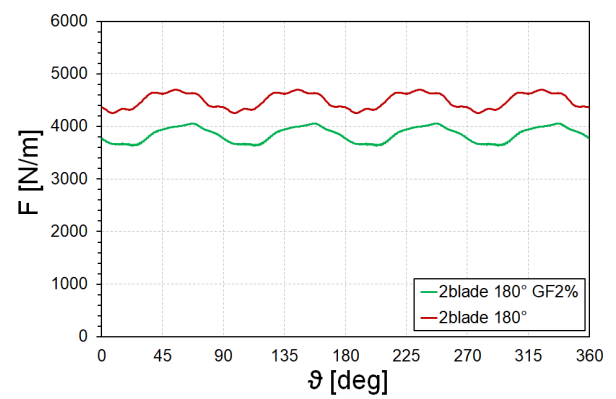

(a)

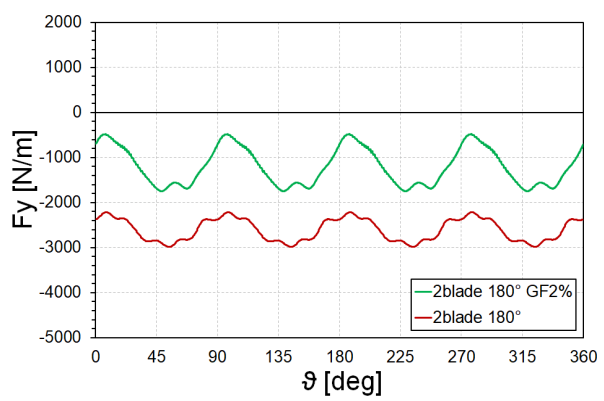

(b)

Fig. 7. (a) global force magnitude and (b) global lateral force per unit span at $T S R_{o p t}$.

Concerning the hydrodynamic loads experienced by the single blade, Figure 8.a reports the trends of normal force per unit span on a single blade during its revolution for the tested $2 B-H$ cases at each respective optimal TSR. From a perusal of the figure, it can be readily seen that negative peak of normal force is reduced by $13 \%$, thanks to the lower lift generated during the upwind half of the cycle. Again, such result represents a great advantage for the mechanical resistance of the machine, since the hydrodynamic forces are more relevant than centrifugal forces.

The relative velocity contours of Figure 8.b allow explaining the fluid-dynamic behaviour of the Gurney Flap. In case of negative incidence $\left(\vartheta=90^{\circ}\right.$, upwind half), the GF operates in the separated region of the suction side of the blade, leading to a worsening of the lift and a higher drag resistance. Conversely, in case of positive incidence $\left(\vartheta=270^{\circ}\right.$, downwind half), the GF operates in the pressure side of the blade, thus providing a substantial lift increase, even if in case of small values of the incidence angle.

Finally, the evolution of the turbine's wake for the $2 B-H$ and $2 B-H G F 2 \%$ cases at each respective optimal TSR is shown in Figure 9. The wake of the $2 B-H$ case is more asymmetrical, since the high deficit region is shifted toward the leeward side (bottom side, in the figure) of the revolution, as well as the acceleration of the absolute flow in the regions external to the rotor is more evident around the $\vartheta=180^{\circ}$ position than the $\vartheta=0^{\circ}$ position. Conversely, the wake of the $2 B-H G F 2 \%$ case is more symmetrical, which is also due to the higher downwind energy extraction, as can be noticed by the increased velocity deficit behind the blade at the $\vartheta=300^{\circ}$ position.

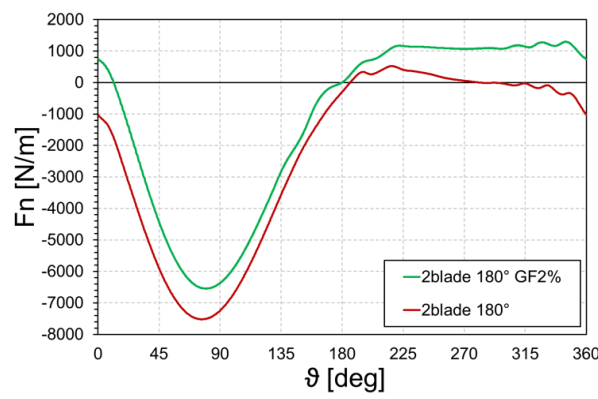

(a)

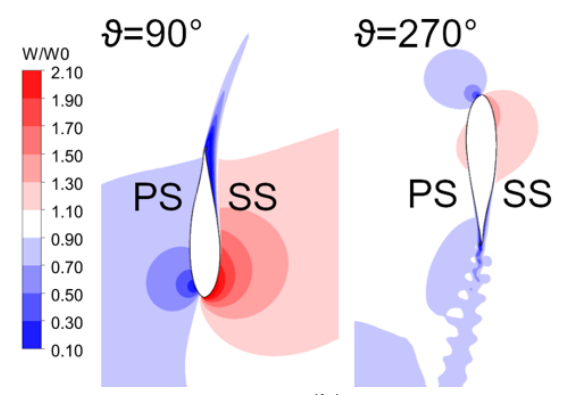

(b)

Fig. 8. (a) normal force of a single blade per unit span at $T S R_{\text {opt }}$, (b) relative velocity contours for the $2 B-H G F 2 \%$ case $\vartheta=90^{\circ}$ and at $\vartheta=270^{\circ}$. 


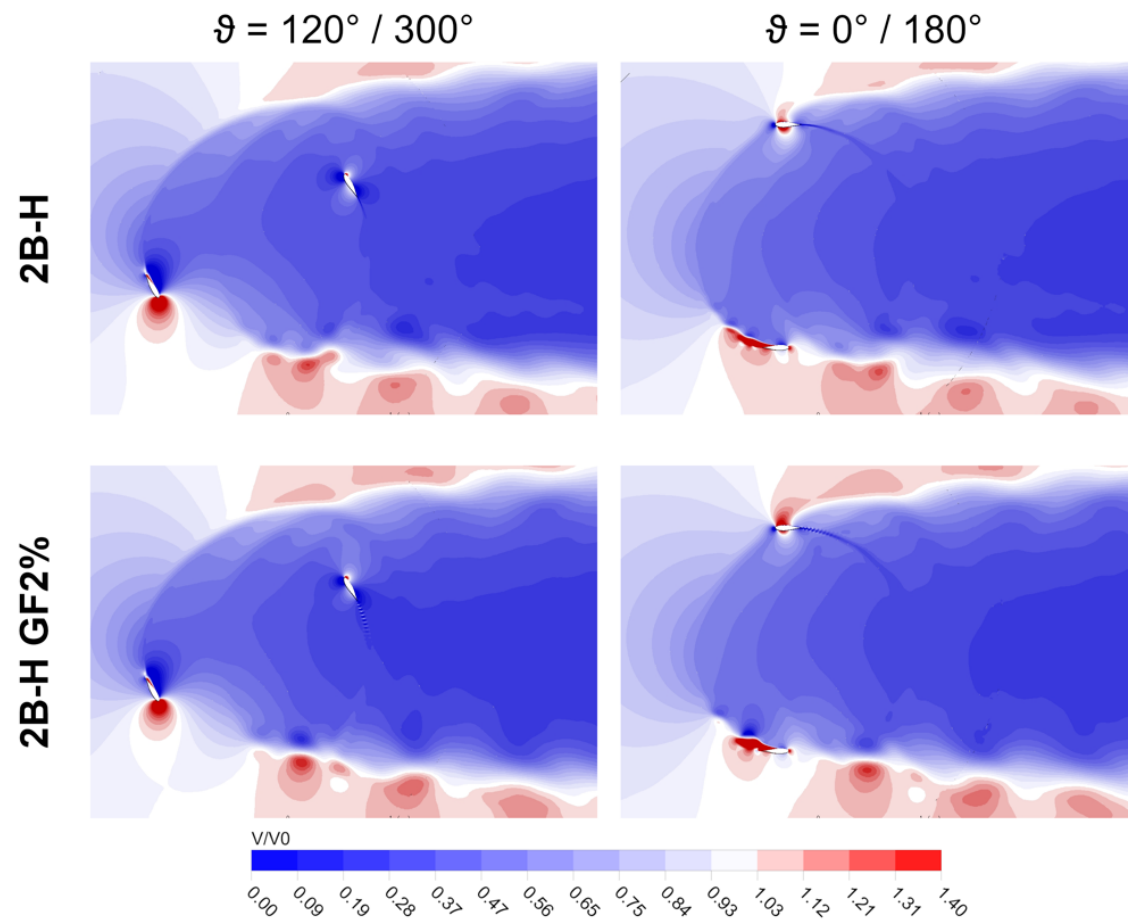

Fig. 9. Absolute velocity contours at $T S R_{\text {opt }}$.

\section{Conclusions}

In the present study, a design adaptation of an industrial study case application of a standard vertical axis Darrieus rotor to operate in a water flow via hydrokinetic technology has been shown. High-fidelity CFD simulations were carried out in order to numerically evaluate the influence of the rotor arrangement and the use of trailing edge tabs on the expected power performance as well as on the blade and turbine hydrodynamic loadings.

Concerning the effect of different rotor arrangements, the results clearly showed that the two-blade rotor configurations allow increasing the power output by roughly $13 \%$ at the expenses of a $23.5 \%$ increase of the optimal operating TSR, as could be expected thanks to a lower solidity rotor. In addition, the mid span blade splitting to position the upper and bottomer portions perpendicularly to each other allowed a strong reduction of the global force oscillations, thus ensuring a more balanced design and, therefore, a longer life expectancy of the machine.

The two blade $\mathrm{H}$-type rotor, namely $2 B-H$, was then selected for analysing the influence of installing a Gurney Flap with a height of $2 \% c$ on the inner side of the blade. The use of a passive flow control device was found to be helpful in reaching the best trade-off between performance increase and limitation of the structural loadings. Indeed, the maximum power was increased by $10.7 \%$ (slightly lower gain than the corresponding smooth case), but such result was achieved with much more favourable conditions in terms of structural stress, both related to fluid dynamic loadings and inertial loadings:

- regarding the inertial loads, the power curve of the $2 B-H G F 2 \%$ configuration was shifted towards lower rotating speeds, with a $9.5 \%$ reduction of the optimal TSR respect to the smooth $2 B-H$ configuration, thus guaranteeing lower centrifugal forces; 
- regarding the hydrodynamic loads, the global lateral force acting on the central shaft of rotor was reduced by roughly $50 \%$, thus leading to an average force being $14 \%$ lower, and the negative peak of normal force acting on a single blade was reduced by $13 \%$ thanks to the lower lift generated during the upwind half of the cycle.

\section{Nomenclature}

$\begin{array}{ll}C F D & \text { Computational Fluid Dynamics } \\ c & \text { blade chord, m } \\ C_{P}, C_{T} & \text { power, torque coefficient } \\ D & \text { rotor diameter, } \mathrm{m} \\ F_{N} & \text { blade normal force } \\ F_{X}, F_{Y}, F & \text { rotor axial, lateral and global force } \\ G F & \text { Gurney Flap } \\ H & \text { rotor height, } \mathrm{m} \\ H K T & \text { Hydrokinetic Turbine } \\ N & \text { number of rotor blades } \\ P S & \text { Pressure Side } \\ R & \text { turbine radius, } \mathrm{m} \\ R e_{c} & \text { chord-based Reynolds number } \\ S S & \text { Suction Side } \\ T S R & \text { Tip-Speed Ratio } \\ V_{0} & \text { freestream velocity, ms }{ }^{-1} \\ V A W T & \text { Vertical Axis Wind Turbine } \\ U R A N S & \text { Unsteady Reynolds-Averaged Navier-Stokes equations } \\ W & \text { relative velocity, ms }{ }^{-1} \\ W T & \text { Wind Turbine } \\ \vartheta & \text { blade azimuthal position, deg } \\ \sigma & \text { solidity } \\ \Omega & \text { turbine rotational speed, } \mathrm{s}^{-1}\end{array}$

\section{References}

1. https://www.iea.org/

2. S. Lundin, J. Forslund, A. Goude, M. Grabbe, K. Yuen, M. Leijon, J. Renew. Sustain. Energy 8, 064501 (2016)

3. G. Saini, R. P. Saini, Int. J. Energy Res. 43, 6639 (2019)

4. D. De Tavernier, C. Ferreira, G. Van Bussel, Wind Energy 22, 547 (2019)

5. A. Rezaeiha, H. Montazeri, B. Blocken, Energy 165, 1129 (2018)

6. A. Hunt, C. Stringer, B. Polagye, J. Ren. and Sust. Energy 12, 054501 (2020)

7. B. Paillard, J. A. Astolfi, F. Hauville, Int. J. Mar. Energy 11, 9 (2015)

8. I. S. Hwang, Y. H. Lee, S. J. Kim SJ, Applied Energy 86, 1532 (2009)

9. M. Alidadi, V. Klaptocz, G. W. Rawlings, Y. Nabavi, S. Calisal, J. Offshore Mech. Arct. Eng. 134, 021201 (2012)

10. R. H. Liebeck, Journal of Aircraft 15, 547 (1978)

11. Y. Li, J. Wang, P. Zhang, Flow Turbul. Combust. 68, 27 (2002)

12. L. Brown, A. Filippone, Aeronaut. J. 107, 539 (2003) 
13. A. Bianchini, F. Balduzzi, D. Di Rosa, G. Ferrara, Energy Conversion and Management 184, 402 (2019)

14. P. Wiśniewski, F. Balduzzi, Z. Buliński, A. Bianchini, Energies 13, 1877 (2020)

15. F. Balduzzi, D. Holst, P. F. Melani, F. Wegner, C. N. Nayeri, G. Ferrara, C. O. Paschereit, A. Bianchini, J. of Eng. for Gas Turbines and Power 143, (2021)

16. F. Balduzzi, A. Bianchini, R. Maleci, G. Ferrara, L. Ferrari, Renewable Energy 85, 419 (2016)

17. A. Bianchini, F. Balduzzi, P. Bachant, G. Ferrara, L. Ferrari, Energy Conversion and Management 136, 318 (2017)

18. F. Balduzzi, A. Bianchini, G. Ferrara, L. Ferrari, Energy 97, 246 (2016) 